\title{
ACONDICIONAMIENTO FÍSICO EN EL MEDIO ACUÁTICO
}

\author{
Pablo Jorge Marcos-Pardo \\ Universidad Católica de Murcia (España)
}

\section{OPEN ACCESS}

Correspondencia

Pablo Jorge Marcos-Pardo

Facultad del Deporte

Universidad Católica de Murcia (UCAM) Av. de los Jerónimos, 135, 30107,

Murcia (Spain) pmarcos@ucam.edu

Citación:

Marcos-Pardo, P. J. (2019). Acondicionamiento físico en el médio acuático. RIAA. Revista de Investigación

en Actividades Acuáticas, 3(5), 1-2. https://doi.org/10.21134/riaa.v3i5.1634

@creative

(c) (1)(2)(2)

Creative Commons Licens Esta obra está bajo una licencia de Creative Commons ReconocimientoNoComercial-Compartir-Igua 4.0 Internacional
Hablar de acondicionamiento físico en el medio acuático implica definir diversos términos como actividad física, ejercicio físico, condición física y estilo de vida por su estrecha relación con la salud. Se define actividad física (AF) como cualquier movimiento corporal producido por los músculos esqueléticos que requieren de un gasto energético; ejercicio físico (EF) es un tipo de actividad física en la que hay movimientos corporales repetitivos, planificados y estructurados, y que se hacen con un objetivo para mejorar o mantener uno o más componentes de la condición física (CF) (Caspersen, Powell, \& Christenson, 1985). Se denomina estilo de vida al conjunto de hábitos cotidianos de comportamiento de una persona con los factores ambientales. Se asocia directamente el estado de salud con el estilo de vida saludable. La AF y el EF a través del acondicionamiento físico es parte integral de cualquier plan para asegurar a las personas una vida saludable. Las personas deben integrar en su vida unos hábitos saludables que le permitan facilitar la pérdida de peso, disminuir la presión arterial, combatir las dislipidemias y en términos generales, reducir el riesgo de enfermedades crónicas y de todas las causas de mortalidad. La Organización Mundial de la Salud (OMS, 2002), define el término salud como "el estado completo de bienestar físico, mental y social y no la mera ausencia de enfermedad".

La investigación científica viene demostrando que el EF tiene un alto poder en la prevención y tratamiento de enfermedades físicas y mentales, que están afectando a la sociedad actual. Diversas investigaciones apuntan que el EF influye en la prevención y tratamiento de enfermedades crónicas derivadas del sedentarismo. En los últimos diez años, más de 30.000 publicaciones en revistas de impacto avalan los beneficios de un adecuado nivel de condición física (CF) para la salud (Del Valle, 2010).

Las personas que son activas físicamente tienden a desarrollar y mantener altos niveles de CF, también conocido como "physical fitness" o fitness. La investigación epidemiológica ha permitido demostrar a través de diversos estudios el efecto protector que tiene el fitness sobre distintas enfermedades crónicas como osteoporosis, enfermedad coronaria, hipertensión, colesterol, diabetes mellitus, cáncer de colon, ansiedad, depresión, trastornos mentales entre otras (Bouchard, Depres, \& Tremblay, 1993; ACSM, 1993, 2009). Los bajos niveles de actividad física aumentan todas las causas de mortalidad; el aumento en la AF y en la práctica de EF durante la edad adulta se asocia con disminución en el riesgo de mortalidad.

Según el INE (2009), la incidencia de enfermedades crónicas detectadas en la población española mayor de 15 años es elevada. Tales enfermedades son principalemente la hipertensión arterial, la artrosis, hipercolesterolemia, la depresión, ansiedad y otros trastornos mentales. Encontramos evidencia científica de que las personas que mantienen o aumentan parámetros de su CF, son capaces de desarrollar mayor calidad de vida, desarrollan menos enfermedades crónicas; mejoran el equilibrio, la coordinación, la agilidad y se previene el riesgo de caídas en los mayores. La gente más activa desarrolla menos enfermedad que los menos activos, y si las sufren, serán menos severas y se presentarán a mayor edad.

Se define acondicionamiento físico en el medio acuático como ejercicios de acondicionamiento físico realizados en el medio acuático, habitualmente en posiciones verticales y que utilizan la resistencia del agua, con una orientación hacia la diversión, el bienestar saludable o la terapia (Colado, 2004). El acondicionamiento físico en el medio acuático traducido como EF regular, se considera como un componente importante de los denominados estilos de vida saludables. Es de anotar que se considera al ser humano como el único animal que necesita acondicionamiento físico y el medio acuático ofrece unas características especiales que no ofrece el terrestre (hipogravidez, presión hidrostática, resistencia al movimiento, termoregulación, etc.). La persona pesa menos, se encuentra más cómoda, si sufre una caída hay menor riesgo de lesión, un ejercicio es capaz de entrenar musculatura agonista y antagonista a la vez debido a la resistencia del agua, se favorece el retorno venoso, entre otros muchos beneficios. 
El ejercicio físico a través del acondicionamiento físico en el medio acuático debe estar incluido en el estilo de vida de una persona como parte de sus hábitos diarios, pero se debe realizar bajo la supervisión de un experto cualificado, que prescriba y lo diseñe indicando la intensidad, el tipo de ejercicios, el tiempo de práctica, la frecuencia semanal o diaria y la progresión, y que cumpla con los requisitos mínimos para que su práctica ofrezca efectos beneficiosos para la salud del practicante. Lo anterior implica que el ejercicio físico deberá formularse, como un medicamento más que recibirá la persona y que en muchos casos con la dosis adecuada, evitará el consumo de medicación y de recursos médicos. Las políticas de salud pública en los países industrializados, han definido como prioritario los programas de ejercicio físico preventivo, porque han visto la necesidad de incorporarlo oficialmente en la vida diaria.

Se puede concluir diciendo que el acondicionamiento físico debe ser parte de los hábitos saludables diarios, siendo el medio acuático un entorno eficaz y seguro para cualquier persona y que le ofrece unas posibilidades que el medio terrestre no puede. Las personas deben adoptar un programa de ejercicio de acuerdo con sus necesidades y su tiempo libre, teniendo en cuenta que por lo menos 30 minutos del día se reserven para hacer ejercicio a intensidade moderada y se eviten actividades que promuevan el sedentarismo.

\section{Referencias}

American College of Sports Medicine. Position standard (1993). Physical activity, physical fitness and hypertension. Medicine \& Science in Sports \& Exercise, 10.

American College of Sports Medicine. Position standard (2009). Progression models in resistance training for healthy adults. Medicine \& Science in Sports \& Exercise, 41.

Bouchard, C., Depres, J. P., \& Tremblay, A. (1993). Exercise and obesity. Obesity Research, 1, 133-147.

Caspersen, C. J., Powell, K. E., \& Christenson, G. M (1985). Physical activity, exercise and physical fitness. Public Health Reports, 100, 125-131.

Colado, J. C. (2004). Acondicionamiento físico en el medio acuático. Barcelona: Paidotribo.

Del Valle, M. (2010). Ejercicio físico y salud. ¿Dónde estamos? Archivos de Medicina del Deporte, 139(27), 327-328

Instituto Nacional de Estadística (INE) (2009). Salud. Cifras INE. Boletín Informativo del Instituto Nacional de Estadistica, 2, 1-12.

Organización Mundial de la Salud (2002). The World Health Report: reducing risks, promoting healthy life. Geneva: World Health Organization. 